\title{
III. Der galvanische Pinsel. Die Behandlung der Impotenz, Ischias und Tabes dorsalis.
}

\author{
Von Dr. A. Witkowski in Berlin.
}

Wahrend der faradische Pinsel seit Jahrzehnten in die Elektrotherapie eingeführt ist, findet der galvanische Pinsel nur wenig Verwendung. Wiesner ${ }^{3}$ ) empfahl ihn 1868 bei Behandlung einer Trigeminusneuralgie, Seeger $\left.{ }^{4}\right) 1872$ bei Ischias. Erb erwähnt in seinem Handbuch der Elektrotherapie den mit der Kathode armirten Metallpinsel gegen Anästhesie, und Seeligmüiler ${ }^{5}$ ) hat 1883, dann Böttger6) 1884 auf seine Veranlassung den galvanischen Pinsel bei chronischem Gelenkrheumatismus in Anwendung gebracht. Seitdem ist es still geworden von dem galvanischen Pinsel; denn man kann nicht sagen, dass die genannten Empfehlungen Anklang bei den Aerzten gefunden hätten.

Die Wirkung des faradischen Pinsels ist eine erregende, eine irritative. Die gepinselte Haut wird zuerst blass, dann röthet sie sich, und es tritt Schmerzempfindung auf, je nach der Stromstärke grösser oder geringer. Der galvanische Pinsel übt dieselbe Wirkung aus: Blässe, Röthung, Schmerz. Ein Vergleich des Grades und der Dauer der einzelnen Stadien zwischen beiden Stromarten ist ungemein schwierig, da es einen absoluten Gradmesser für den faradischen Strom nicht giebt. Ich habe hierzu die Reizwirkung auf einige besonders bequem liegende Nervenstämme benutzt, fǜ die Unterextremitäten auf den Nervus peroneus, für die Oberextremitäten auf den Nervus ulnaris und habe diejenigen Ströme als gleich stark angesprochen, die ungefähr gleich grosse Muskelzuckungen verursachten. Hiernach ergab sich eine grosse Mannigfaltigkeit in den Reactionsverhältnissen der Haut, auf die ich hier nicht näher eingehen will; constant nur schien bei dem galvanischen Pinsel die Röthe intensiver und andauernder zu sein; sicher aber ruft der galvanische Pinsel eine weit grössere Schmerzempfindung hervor, als der faradische. Es ergiebt sicli also aus diesen Versuchen die Ueberlegenheit des galvanischen Pinsels in der Reizwirkung: Röthe-Schmerz. Und hierauf kommt es einzig und allein an.

Die ganze therapeutische Aufgabe des faradischen Pinsels besteht in der Reizung der Haut, sei es nun behufs direkter Beeinflussung derselben, sei es behufs Ausübung des sogenannten Gegellreizes, sei es behufs reflektorischer Fernwirkung auf die nervösen Centralorgane. Ausser der genannten Wirkung wird man aber dem galvanischen Pinsel noch diejenige zusprechen müssen, die dem galvanischen Strome überhaupt vor dem faradischen eigen ist, nämlich die Wirkung in die Tiefe. Der faradische Strom hat nur Oberflächenwirkung; will man Stromschleifen direkt in die tiefer gelegenen Theile gelangen lassen, so muss man sich des galvanischen Stromes bedienen. Aus dem Gesagten ergiebt sich, dass der galvanische Pinsel den faradischen quantitativ und qualitativ in der Wirkung ïberragt.

Der Grund, weshalb trotzdem der galvanische Pinsel bisher so wenig Beachtung gefunden hat, liegt in den gefürchteten Folgen seiner Behandlung. Zunächst bemerke ich, dass mit dem Absetzen der Elektroden bei beiden Stromarten der Schmerz sofort aufhört; die Röthe dagegen hält sich noch eine Zeit lang, beim galvanischen

1) Dr. Giusti, Un caso di tetano curato con siero antitetanico. Gazzetta degli Ospitali 1894.

2) Escherich, Vier mit Tizzoni's Antitoxin behandelte Fälle von Trismus et Tetanus neonatorum. Wiener klin. Wochenschr. 1893, No. 32 :

$\left.{ }^{3}\right)$ Wiesner, Zwei Fälle von Heilung schwerer und langwieriger Prosopalgie durch den constanten Strom. Berl. klin. Wochenschr. 1868; No. 17, 18.

4) Se eger, Abhandl. über Neuralgie, bezw. Ischialgie. Wien. med. Presse 1872, No. $34,35,37,38$.

5) Seeligmuller, Naturforscherversammlung in Freiburg 1883.

6) Böttger, Beitrag zur Behandlung des chronischen Gelenkrheumatismus mit Elektricität. Inaugural-Dissert., Halle 1884. 
Strom viel länger, als beim faradischen. Erb ${ }^{1}$ ) hat bei ersterem noch nach 6-10 Tagen bei gewissen Einwirkungen auf die Haut, z. B. nach Gebrauch eines Dampfbades, die Applicationsstellen sich wieder röthen sehen. Zum Unterschied aber von dem faradischen bewirkt der galvanische Pinsel, wenn er längere Zeit auf einer Stelle haftet, Aetzungen und Verschorfungen. Seeligmüller benutzte diese Aetzwirkung therapeutisch bei Gelenkleiden. Dies würde nur eine Fehlerwirkung sein, wie sie den meisten der zur Reizung der Haut im Gebrauch befindlichen Mittel, der ganzen Gruppe der Vesicantien u. a. m., ebenfalls anhaftet. Aber man kann dieselbe sehr leicht dadurch vermeiden, dass man den Pinsel häufig von der behandelten Stelle abhebt und ausserdem die Stelle leicht anfeuchtet. - Ein zweiter Vorwurf, den man dem galvanischen Pinsel gegenüber dem faradischen macht, ist die heftige Schmerzerregung. Dieser Vorwurf gleicht ungeführ dem, den man dem Chloroform machen kann, weil es betäubt. Da der galvanische Pinsel zur Reizung der sensiblen Hautnerven angewandt wird, so kann die gewollte Wirkung nur die des Schmerzes sein. Und dass man den galvanischen Strom bei einem einigermaassen guten Apparate genau dosiren kann, bedarf keiner Erläuterung.

Unangenehm ist bei längerem Hin- und Herstreichen nur die Wirkung des rauhen Metallfadens auf die Haut, eine Wirkung, die sich aber sowohl beim faradischen, wie beim galvanischen Pinsel äussert. Diesem Uebelstande begegne ich dadurch, dass ich den Pinsel kurz, bei längerem Gebrauche öfter in kaltes Wasser tauche, so dass nur einige Tropfen an den Fäden hängen bleiben. Es ist richtig, dass die Oberflächenwirkung des galvanischen Stromes durch Anfeuchtung der Haut verringert wird, da hierdurch der Widerstand der Haut vermindert wird. Aber die erwähnte Art der Anfeuchtung ist so gering, dass sie kaum für die Widerstandsbrechung in Betracht kommt, und doch gross genug, um Verletzungen der Haut durch den rauhen trockenen Metallfaden zu verhindern.

Wir besitzen somit im galvanischen Pinsel einen den faradischen Pinsel an Intensität weit überragenden, acut wirkenden Hautreiz, den man ganz genau lokalisiren und dosiren und den man beliebig oft zur Anwendung bringen kann, ohne irgend welche schädlichen Nachwirkungen zu verursachen.

In dreierlei Weise dient der Hautreiz zur Behandlung von Krankheiten :

1. Der Hautreiz dient zur direkten Erregung der sensiblen und vasomotorischen Apparate der Haut. Das Behandlungsobject ist die periphere Anästhesie und Parästhesie einerseits, Circulationsstörungen (mangelhafte Ernährung) andererseits. Behufs Behandlung der ersten Gruppe wird der trockene, mit der Kathode armirte Metallpinsel auf die erkrankte Stelle aufgesetzt. Er bleibt so lange haften, bis heftige Schmerzempfindung entsteht. Alsdann wird er abgehoben, um nach kürzester Frist (einige Secunden) wieder in gleicher Weise aufgesetzt zu werden. Die ganze Procedur dauert zwei bis drei Minuten. In denjenigen Fällen, in denen vollkommene Anästhesie besteht, bleibt der Pinsel jedesmal ca. 10-15 Secunden haften.

Bei der zu zweit genannten Gruppe handelt es sich um die Behandlung partieller Anämie. Die betreffende Stelle wird mit dem in oben erwähnter Weise leicht angefeuchteten Kathodenpinsel längere Zeit, meist bis intensive Röthung eingetreten ist, gepinselt.

Es giebt eine grosse Reihe von Fällen, in denen die Herabsetzung der Sensibilität mit Anämie gepaart ist.2) Ich greife von diesen Fällen die durch Onanie verursachte Impotenz heraus. Es handelt sich um eine Erschöpfungsneurose, deren Ursache in erster Reihe in peripherer Ueberreizung zu suchen ist. Die Prognose ist nicht sehr günstig. Ich empfehle die folgende Methode. Grundbedingung eines Erfolges ist vollkommene Enthaltsamkeit von jeder geschlechtlichen Aufregung. Die direkte, auf Reizung der erschlafften peripheren Organe gerichtete Behandlung besteht darin, dass eine gut angefeuchtete, kleine, mit der Kathode armirte Metallplatte auf verschiedene Theile des Gliedes aufgesetzt wird und durch häufige Oeffnung des starken Stromes Reizung bewirkt wird. Alsdann wird der mit einem schwächeren Strome armirte, leicht angefeuchtete Kathodenmetallpinsel längs des Gliedes ein paar mal hin- und hergeführt, während er auf die Glans in oben genannter Weise, aber leicht angefeuchtet, wiederholt bis zur Schmerzempfindung aufgesetzt wird. Die Procedur dauert drei Minuten und wird zweitäglich wiederholt. Nach Ablauf von drei Wochen werden der Behandlung protahirte Kohlensäurebäder zugefügt, wie sie in Berlin in einigen Anstalten nach dem Lippert'schen System in vorzüglicher Weise verabreicht werden. Die Dauer eines Bades muss $1 / 2-3 / 4$ Stunde betragen. Es werden durchschnittlich 15 Bäder

1) Handbuch der Elektrotherapie. Tabes.

2) Siehe die folgende Besprechung des Zusammenhanges bei der zweitäglich genommen; an den Zwischentagen wird elektrisirt. Nach Ablauf dieser Cur wird eine Pause von zwei Monaten in der Behandlung gemacht. Alsdann wird die Cur in milderer Form durchschnittlich zweimalige Behandlung in der Woche - wiederholt. Mit dieser Methode habe ich selbst in veralteten Fällen noch gute Resultate erzielt.

2. Der Hautreiz dient als Gegenreiz. Weshalb bei Reizung der Haut tiefer gelegene erkrankte Theile, wie Muskeln und Nerven, die sich im Reizzustand befinden, heilen, ist nicht recht klar. Aber der häufig eclatante Erfolg beweist die Richtigkeit der Behandlung. $\mathrm{Zu}$ dieser Gruppe gehört das ganze Gebiet der Myalgieen, Neuralgieen, Arthralgieen. Ich wende bei allen diesen Fällen den galvanischen Pinsel an, da er den intensivsten Hautreiz darstellt, der am leichtesten abstufbar, genau begrenzt und ohne Nachempfindung und schädliche Folgen für die Haut anwendbar ist. Die Methode ist Pinselung der erkrankten Stelle mit dem leicht angefeuchteten Pinsel.

Zur Einzelbesprechung greife ich die Ischias heraus. Bei der Behandlung dieser Krankheit tritt die Ueberlegenheit des galvanischen Pinsels über die anderen elektrischen Methoden besonders hervor. Der Patient liegt auf dem Bauch. Die Haut wird über dem Ischiadicus in seiner ganzen Ausdehnung von seinem Austritt bis zu den letzten Ausläufern mit dem leicht angefeuchteten Kathoden* pinsel bei starkem Strome ca. fünf Minuten lang kräftig gepinselt. Währenddem haftet der Pinsel wiederholt auf den Schmerzpunkten kurze Zeit. Mit dieser täglich oder zweitäglich zu wiederholenden Behandlungsart erreicht man oft glänzende Erfolge. Viele alte Fälle, die anderen Methoden Widerstand leisteten, heilten, und zwar in kürzester Zeit. Aber nicht immer gelingt dies. Trotzdem Besserung in allen Fällen zu erzielen ist, geht dieselbe manchmal über einen bestimmten Punkt nicht hinaus; die Heilung bleibt aus. Um diese zu erreichen, empfehle ich die Combination dreier bekannter Methoden: Massage — galvanische Pinselung - Nervendehnung.

Während der Patient auf dem Bauche liegt, wird das ganze Gebiet des Nervus ischiadicus ca. fünf Minuten lang kräftig massirt. Nachdem das zur Massage benutzte Fett sorgfältig abgerieben ist, folgt wiederum ca. fünf Minuten lang die oben beschriebene galvanische Pinselung. Es ist selbstverständlich, dass der Pinsel bei so langer Anwendung häufig angefeuchtet werden muss, wenn auch nur durch kurzes einmaliges Eintauchen in kaltes Wasser. Nach Beendigung der Pinselung legt sich der Patient auf den Rücken. Der Arzt greift mit der einen Hand unter den Hacken des erkrankten Beines, mit der anderen drückt er fest auf das Kniegelenk. Alsdann hebt er plötzlich und mit grosser Kraft das in der Streckung gehaltene Bein an, um eine möglichst ergiebige Beugung in der Hüfte auszuführen. Die Bewegung wird in ganz kurzen Zwischenpausen vier- bis fünfmal wiederholt. Die Methode, die im Princip schon lange als unblutige Nervendehnung bekannt ist, ist jetzt von Bonuzzil) und später von Benedikt²) wieder neu entdeckt worden. So leicht wie die Verfasser sich die Beugung der Hüfte denken, ist dieselbe wenigstens bei an Ischias Leidenden nun nicht. Die Kranken spannen, durch den heftigen Schmerz veranlasst, die Hüftmuskeln auf das äusserste an, um die Beugung $\mathrm{zu}$ verhindern.

Auch halte ich behufs Nervendehnung die Beugung nicht für genügend, wenn dabei das Bein nicht energisch gestreckt wird. Beide Indicationen erfüllt die oben angeführte Methode leicht und schnell, was bei der Schmerzhaftigkeit der Affection ein nicht zu unterschätzender Vortheil ist.

Von den drei zur Behandlung angegebenen Acten - die in ganz schweren Fällen täglich, sonst zweitäglich ausgeführt werden müssen - halte ich die Massage für den direkt am wenigsten wirksamen. Doch möchte $\cdot$ ich sie als Hülfsmittel zur Kräftigung der bei längerer Dauer der Krankheit stets in Mitleidenschaft gezogenen Muskeln nicht entbehren. Das Hauptgewicht lege ich auf die galvanische Pinselung und die Nervendehnung. So ungemein schmerzhaft diese beiden Acte der Behandlung auch zu sein pflegen, so entziehen sich die Patienten derselben doch niemals. Denn der Erfolg ist nach jeder einzelnen Vornahme ein ganz evidenter. Patienten, die zur Behandlung getragen werden müssen, können nach einer Sitzung häufig schon eine Zeit lang gehen. Allerdings pflegt der Erfolg in seinem ganzen Umfange nur eine Reihe von Stunden anzuhalten. Aber es bleibt nach jeder Sitzung doch immer ein gut Theil Besserung dauernd übrig. Führt man die Behandlung nun regelmässig mit nur 24 stündigen Pausen durch, so erreicht man in kürzester Zeit vollkommene Heilung. Ich

1) Bon uzzi, Atti della R. Accademia medica di Roma 1890 91, S.257. Referat in Eulenburg's Realencyclopädie Bd. 24, S. 649.

2) Benedikt, Die Methode Bonuzzi. Die Behandlung der Tabes. Wiener med. Presse 1892, No. 1. 
habe Patienten, die Jahre lang an schwerster Ischias, complicirt mit hochgradiger Muskelatrophie, litten, in vier bis sechs Wochen durch die angegebene combinirte Behandlungsmethode geheilt.

3. Der Hautreiz dient zur Fernwirkung auf die nervösen Centralorgane (reflectorische Wirkung). Nothnagel ${ }^{1}$ ) fand, dass Hautreizung Verengerung der Hirngefässe bewirkt. Rumpf ${ }^{2}$ ) hat dann nach gewiesen, dass genügend starke faradische Reizung einer Extremität bei Fröschen und Kaninchen Erweiterung der Piaarterien auf der entgegengesetzten Hirnhemisphäre hervorruft. Löwenfeld ${ }^{3}$ ) hat dann gefunden, dass starke faradische Hautreizungen Erweiterung der Piagefässe im Rückenmark bewirken Auf diese Experimentalerfolge gestützt, hat man nun eine Behandlungsart von Krankheiten der Centralorgane, vorzügrlich der Tabes dorsalis gegründet. Durch faradische Pinselung grosser Hautgebiete haben Rumpf und Andere Erfolge bei der letztgenannten Krankheit erzielt.

Bei den Rückenmarkskrankheiten unterscheidet man nicht wie bei den Gehirnkrankheiten direkte und indirekte Symptome. Ich glaube, mit Unrecht. Man würde durch diese Unterscheidung viel genauer die mit Erfolg zu behandelnden Erscheinungen von den incurabeln abgrenzen lernen. Auch bei den chronisch degenerativen Processen, wie bei der Tabes, giebt es indirekte Symptome. Allerdings handelt es sich hierbei nicht um periphere entzündliche oder ödematöse Erscheinungen, wie beim Gehirn. Aber es ist sehr wahrscheinlich, dass bei Ausfall des Nervengewebes an einer Stelle des Rückenmarkes das umliegende Gewebe, des Haltes beraubt, etwas einfällt. Besonders die kleinen Gefässchen werden hierbei leicht einem Drucke unterliegen, gross genug, um die Ernährung und demgemäss die Function der Nervensubstanz zu beeinträchtigen. Hierbei ist das Gewebe anatomisch ganz unverletzt. Wenn es gelingt, die Circulation kräftig anzuregen, den Blutzufluss zu vermehren, die durch den Druck bedingte Anämie zu heben, so werden auch die Krankheitserscheinungen weichen. Die Art der Erkrankung erklärt auch, weshalb die Erscheinungen so leichtem Wechsel unterworfen sind.

$\mathrm{Zu}$ den indirekten Erscheinungen bei der Tabes gehört vorzüglich die gestörte Blasenfunction. Abgesehen von den spontanen Schwankungen, giebt es kaum ein Symptom bei der Tabes, das so leicht sich durch therapeutische Maassnahmen beeinflussen liesse. Allerdings tritt die Wirkung nicht regelmässig ein und ist oft von nicht langer Dauer, aber als Erfolg fast aller Methoden der Behandlung der Tabes wird die Besserung der Blasenstörungen aufgeführt. Auch die neuesten zur Behandlung der Tabes empfohlenen Methoden rühmen dieses Resultat. So erwähnt Eulenb $\mathrm{rg}^{4}$ ) in dem Bericht über die Suspensionsbehandlung in der Eulenbug-Mendel'schen Poliklinik, dass zu den am häufigsten gebesserten Krankheitserscheinungen auch die Blasensymptome gehören, wenn auch nur mit temporärer Wirkung. Jürgensen ${ }^{5}$, der zur Behandlung der Tabes das Tragen von nach dem System Hessing gearbeiteten Stoffcorsetts empfiehlt, meint, dass sich bei dieser Behandlung zunächst die Blasenstörungen bessern. In seinem Artikel über Suggestionstherapie betont v. Corval ${ }^{6}$ ), dass fast alle Beobachter über mehr oder minder entschiedene ... Kräftigung der Blasenschliessmuskel berichten.

Diesen Methoden zur Behandlung der tabischen Blasenstörungen reihe ich die mittels des galvanischen Pinsels an. Sie übertrifft dieselben an Leichtigkeit der Ausführung, Sicherheit, Schnelligkeit und Dauer des Erfolges. Sie wird ausgefüht durch galvanische Pilselung der Haut iiber dem Lendenmark bei angefeuchtetem Pinsel mit starken Strömen, am besten täglich, jedes Mal bis zu intensiver Hautröthung. Die Wirkung beginnt häufig schon nach zwei bis drei Pinselungen, und nach Ablauf einer Woche ist meist das Aufhören der Blasenstörung erreicht. Die Behandlung muss dann noch ein bis zwei Wochen fortgesetzt werden.

Selbst in weit vorgeschrittenen Fällen von Tabes erreicht man noch gute Resultate. Die Dauer dẹr Wirkung beläuft sich auf Wochen, Monate, Jahre. Hierbei kommen eben die direkten, durch die Krankheit selbst im Rückenmark gesetzten Veränderungen wieder in's Spiel, welche natürlich auch wieder Veränderungen der indirekten Symptome veranlassen. Schliesslich werden auch die indirekten Symptome durch Uebergreifen des Processes auf das Blasencentrum zu direkten, und dann hört die Wirksamkeit aller

1) Nothaagel, Die vasomotorischen Nerven der Gehirngefässe. Virchow's Archiv Bd. 40. S. 203, 1867.

2) Rumpf, Deutsche med. Wochenschr. 1881, No. 29, und Archiv f. Psychiatrie 1881 .

3) Löwenfeld, Untersuchungen zur Elektrotherapie des Rackenmarks. München 1883

4) Realencyclopädio Bd. 23, S. 649.

5) Deutsche med. Wochenschr. 1889, No. 40.

6) Realencyclopädie Bd. 23 , S. 637.
Methoden auf. Bis zu diesem Punkte aber gehört die Behandlung mit dem galvanischen Pinsel zu den weitaus wirksamsten. Beim Wiederauftreten der Störungen ist bei der Einfachheit der Methode eine Wiederholung leicht zu bewerkstelligen.

Es ist sehr interessant, die einzelnen Stufen der Wirksamkeit der Behandlung in der subjectiven Empfindung des Patienten zu beobachten. Die Mehrzahl der in meine Behandlung gekommenen tabischen Blasenstörungen kam zustande infolge von Anästhesie. Die Patienten merkten überhaupt nicht, wann die Entleerung der Blase erfolgen sollte. Ein kleinerer Theil bekam allerdings Harndrang, bemerkte es aber zu spät, um noch rechtzeitig zum Abort zu kommen. Alle diese Patienten geben nun an, dass bei der Behandlung mit dem galvanischen Pinsel zuerst die Empfindung des Harndranges auftritt, anfangs kurz vor der Entleerung, dann immer früher und früher, so dass schliesslich den Patienten genug Zeit übrig bleibt, bis zum Abort zu gelangen. Das Gefühl, den Harn besser zurückhalten zu können, haben die Patienten im Anfang der Behandlung nie. In Uebereinstimmung damit bessern sich zuerst die Erscheinungen der Blasenstörung auch nur am Tage, während das nächtliche Bettpissen noch andauert. Es fehlt eben der reflectorische Vorgang des Harndranges und des Zusammenziehens des Schliessmuskels der Blase. Erst nach weiterer Behandlung fühlt der Patient die Möglichkeit, den Harn längere Zeit in der Blase zurückhalten zu können. Es erweitert sich der Zeitraum von der ersten Empfindung des Harndranges bis zu der des Zwanges der Entleerung. Dann hört auch das nächtliche Bettpissen auf.

Ein anderes Symptom, das sich durch die Behandlung leicht beeinflussen lässt und das ich deshalb zu den indirekten zähle, ist die Sensibilitätsstörung der Haut. Es mag auffallen, dass ich eine Erscheinung, auf die viele Forscher eine Theorie von dem Wesen der Tabes gründen, zu den indirekten rechne. Aber es ist unzweifelhaft, dass bis $\mathrm{zu}$ einem ziemlich weit vorgeschrittenen Zeitpunkte der Krankheit die Sensibilitätsstörung zu den wechselnden Symptomen gehört, dass sie demnach bis dahin nicht zu denjenigen Erscheinungen gehören kann, als deren Veranlassung man einen doppelseitigen chronisch degenerativen Process annehmen muss. Sie gehört nicht zu den unwandelbaren Ausfallssymptomen, wie deren eines zum Beispiel der fehlende Patellarreflex darstellt.

Weitere theoretische Betrachtungen führen an dieser Stelle zu weit. Ich beschränke mich im Folgenden auf das Thatsächliche und vor allem auf das für die Behandlung Verwerthbare.

Wenn man die Haut der Unterextremitäten eines Tabikers mit dem galvanischen Pinsel täglich streicht, so bemerkt man, dass der Strom, der im Anfang gar nicht gefühlt wurde, nach einer Reihe von Sitzungen schmerzhaft empfunden wird, häufig derart, dass man genöthigt ist, beträchtlich schwächere Ströme anzu• wellden. Die elektrocutane Sensibilität hat sich eingestellt.

Wenn man die Tast- und Schmerzempfindung an den Unter. extremitaten eines Tabikers vor und nach der galvanischen Pinselung untersucht, so findet man, dass diese Qualitäten nach der Pinselung erhöht, häufig sogar beträchtlich erhöht sind. Nach einem Zeitraum, der nur nach Stunden rechnet, nimmt diese Erhöhung wieder $a b$, aber doch nur so Iangsam, dass am nächsten Tage immer noch ein geringes Mehr an Empfindung übrig geblieben ist. Wiederholt man nun die Behandlung täglich, so kann man es erreichen, dass nach einiger Zeit eine nicht unbedeutende Vermehrung der genannten Empfindungsarten gesichert ist.

Die galvanische Pinselung bewirkt überall auf der Haut Röthung. Aber es fällt auf, dass diese Röthung an Hautstellen, die von Sensibilitätsstörungen ergriffen sind, viel langsamer und schwächer auftritt, ja manchmal überhaupt nur sehr schwer erreichbar ist. Es fällt ferner auf, dass dem Grade der möglichen Hautröthung die Zunahme der Sensibilität zu entsprechen scheint. Ueberall, wo eine beträchtliche Hautröthung gelingt, ist auch die Besserung der Sensibilität eine beträchtliche. Ebenso ist auch im gegentheiligen Falle das Verhältniss ein gleichartiges. Häufig gelingt es erst nach einer grossen Reihe von Sitzungen, eine wesentliche Veränderung in der Möglichkeit der Hautröthung zu bewirken. Bis zu diesem Zeitpunkte muss man dann auch auf den Eintritt einer Besserung der Sensibilitätsstörung warten.

Dies sind die drei Punkte, die sich bei der Behandlung der Unterextremitäten von Tabikern mit dem galvanischem Pinsel auf der Haut bemerkbar machen. Subjectiv macht sich ein wesentlich angenehmeres Gefühl in den Beinen geltend. Die überaus lästige Empfindung der Kälte und der Gefühllosigkeit in den Beinen lässt nach, und in vielen Fällen verringern sich auch die spontanen Schmerzempfindungen nicht unbeträchtlich.

Schon die Möglichkeit, den gerade durch Sensibilitätsstörungen bei Tabikern verursachten qualvollen Zustand $\mathrm{zu}$ bessern, würde die Behandlung mit dem galvanischem Pinsel rechtfertigen. Aber die Wirksamkeit desselben ist damit noch nicht erschöpft. Bei 
längerer Behandlung der Unterextremitäten tritt in der grossen Mehrzahl der Fälle eine Besserung des Ganges ein. Er wird sicherer, und der Patient ist imstande schneller zu gehen, ohne sich der Gefahr des Sturzes anszusetzen. Objectiv lässt sich meist eine Abnalme der Ataxie nachweisen. Die Schwankungen bei Schluss der Augen sind viel weniger ausgiebig als früher. Es gelingt dem Patienten, sich längere Zeit ohne Unterstïtzung der Augen im Gleichgewicht $\mathrm{zu}$ erhalten. $\mathrm{Ob}$ der Grund aller dieser Erscheinungen eimzig und allein in der Besserung der Sensibilitätsstörungen der Haut zu suchen ist, oder ob dabei eine Wirkung auf tiefer gelegene Theile, oder wie für den faradischen Pinsel angenommen wird, eine Fernwirkung auf das Rückenmark selbst noch in Betraclit kommt, darüber will ich mich hier nicht entscheiden. Jedenfalls glaube ich den galvanisclien Pinsel als ein ausgezeichnetes Mittel zur symptomatischen Behandlung des Tabes empfehlen zu dürfen. Die Behandlung führe ich in der Weise aus, dass ich zuerst die Haut über dem Lendenmark mit dem angefeuchteten galvanischen Pinsel bei starkem Strome bis zu intensiver Hautröthung streiche, alsdann die Unterextremitäten in gleicher Weise, jede Extremität 3-4 Minuten lang, möglichst auch bis zur Hautrötliung. Die Behandlung geschielıt am besten täglich, doch gelangt man auch mit zweitägiger Behandlung zun Ziel.

Mit dieser Methode habe iclı zum Theil ansgezeichnete Resultate erzielt. Von Heilung oder Aufhalten der Krankheit ist selbstverständlich gar keine Rede. Nur durch Beeinflussung der indirecten Symptome, d. h. derjenigen, die von in obigem Sinne erkraulkten Nerventheileı, welche also noch nicht der Degeneration verfallen sind, lierrühren, gelingt es eiue Besserung zu erreichen. Die Besserung währt Monate bis Jahre, je nach der Progressivität des anatomischen Processes. Schreitet derselbe Jahre lang nicht fort, so ist es auch leicht, das Bild angeblicher Heilung vorzutäuschen. Hierfür nur ein Beispiel. Ein Locomotivführer kam im Jahre 1888 mit den Zeichen von reflectorischer Pupillenstarre, fehlenden Patellarreflexen, subjectiven und objectiven Sensibilitätsstörungen, Blasenstörungen und Ataxie zu mir. Durch die Behandlung mit dem galvanischen Pinsel sclrwanden die Blasenstörıngen und subjectiven Sensibilitätsstörungen. Die Sicherheit des Ganges stellte sich in bedeutendem Grade wieder ein. Objectiv blieb aber die reflectorisclie Pupillenstarre, die fehlenden Patellarreflexe und geringe Sensibilitätsstörumgen. Auch die Ataxie konnte bei genauer Untersuchung noch nachgewiesen werden. Dieser $\mathrm{Zu}$ stand blieb bis zum Jahre 1891, wo icl den Patienten aus dem Auge verlor. Der Patient hatte das Gefiilll der Heilung, weil er subjectiv nur wenig von seiner Kranklieit merkte.

Sclureitet der anatomische Process fort, so treten naturgemäss Verschlechterungen auf. Man kann dann wiederum versuchen, die indirecten Symptome, falls solche noch vorhanden sind, durch eine Cur zu verbessern. Das ist meiner Meinung nach das höchste, was bei der Behandlung der Tabes überhaupt erreichbar ist, nämlich bei eimem durclı anatomische Verhältnisse gegebenen unabänderlichen Zustande die für den Kranken möglichst günstigsten Bedingungen. 